\title{
Systematische Untersuchungen an Grundwasserkrebsen - eine Bestandsaufnahme \\ (mit der Beschreibung zweier neuer Gattungen der Familie \\ Parabathynellidae, Bathynellacea)
}

von

\author{
Horst Kurt SCHMINKE*
}

\begin{abstract}
SUMMARY
Critical evaluation of recent work on the systematics of subterranean Crustacea (with description of two new genera of Parabathynellidae, Bathynellacea)

This paper reviews critically some recent work on the systematics of subterranean Crustacea, in particular Jakobi's work on the Parastenocarididae (Copepoda), Serban's work on the Bathynellidae (Bathynellacea, Syncarida) and Schminke's revision of the Parabathynellidae (Bathynellacea, Syncarida). It is pointed out that three different approaches have been adopted in each of the three cases and advantages and shortcomings of each of them are analysed. Finally descriptions are given of two new genera of Parabathynellidae, Afrobathynella gen. n. and Nunubathynella gen. n., from a locality near Port Elisabeth (South Africa).
\end{abstract}

\section{EINLEITUNG}

Die Systematik der Grundwasserkrebse ist in den letzten Jahren in eine neue Phase getreten. Die Periode der reinen Bestandsaufnahme und der Zuordnung der aus allen Teilen der Welt bekanntgewordenen Formen zu einigen wenigen Großgattungen neigt sich ihrem Ende entgegen. Statt dessen wird immer häufiger der Schritt vom Stadium allumfassender Gattungen zu dem ihrer Auflösung in ein System geschlossener Verwandtschaftsgruppen gewagt.

Leider wird häufig unterschätzt, welche ausschlaggebende Bedeutung gerade dieser Schritt dafür hat, wie schnell man fortan zu einem tieferen Verständnis der betreffenden Organismengruppe selbst vorstoßen kann, und wie groß der Nutzen sein wird, den man aus der weiteren Beschäftigung mit ihr für die Klärung allgemeinbiologischer Fragestellungen zu ziehen vermag. Voreilig und leichtsinnig getan, kann ein solcher Schritt eine ganze Organismengruppe auf

* Zoologisches Institut der Universität, D-23 Kiel, Hegewischstr. 3. 
Jahre hinaus für die Bearbeitung übergreifender Problemstellungen - mögen sie nun einen ökologischen, zoogeographischen, vergleichend-physiologischen oder dergleichen allgemeinbiologischen Schwerpunkt haben - uninteressant und unbrauchbar machen. Es kann also nicht gleichgültig sein, wie dieser Schritt ausfällt.

Von den Arbeiten der letzten Jahre über Grundwasserkrebse, die deshalb zu erörtern wären, soll nur auf einige eingegangen werden. Bestandsaufnahme wird hier nicht als bloßes Aufzählen und Referieren des Erreichten verstanden, sondern als kritische Auseinandersetzung mit einigen ausgewählten $\mathrm{Ar}$ beiten. Es liegt auf der Hand, daß dafür nur solche in Frage kommen, die Gruppen gewidmet sind, mit denen ich mich selbst befasse oder die ich doch aus eigener Anschauung einigermaßen beurteilen zu können glaube. Im Mittelpunkt der folgenden Erörterungen werden deshalb Jakobis Bearbeitung der Copepodenfamilie Parastenocarididae (1972a), Serbans Arbeiten über die Bathynellidae, die er allein $(1972,1973 \mathrm{a}, \mathrm{b})$ oder in Zusammenarbeit mit anderen Autoren (Serban, Coineau, Delamare Deboutteville, 1972; Delamare Deboutteville \& Serban, 1973) verfaßt hat, und die eigene Revision der Familie Parabathynellidae (Schminke, 1973b) stehen.

\section{PARASTENOCARIDIDAE}

Jakobi (1972a) gibt an, sich bei seiner Gattungseinteilung der Familie Parastenocarididae an den Endopoditen des 4. Peraeopoden der Männchen (Enp. P4 $\hat{o}$ ) gehalten zu haben. Dabei handelt es sich um einen sehr vielgestaltigen, innerhalb der Art jedoch formkonstanten Anhang, an dem sich leicht erkennen läßt, ob man eine neue oder eine schon bekannte Art vor sich hat, und er ist deshalb auch durchweg gut beschrieben und abgebildet worden. Die übrigen Körperanhänge erfuhren dagegen eine sehr unterschiedliche Beachtung. Manche Beschreibungen machen den Eindruck, als behandelten sie fossiles Material, von dem nur mal die eine oder andere Struktur als Abdruck erhalten geblieben ist. Diese Tatsache bestärkte Jakobi in seiner Auffassung, daß allein durch die konsequente Auswertung der Formenvielfalt des Enp. P4 $\hat{\delta}$ Aufschluß über die Verwandtschaftsverhältnisse innerhalb der Familie zu erhalten wäre. Als zusätzliche Gründe für seine Festlegung auf den Enp. P4 $\delta$ nannte er später (1972b, p. 247):

1. 'Er ist der am meisten chitinisierte und morphologisch differenzierte Körperteil,

2. Seine Prägung zeigt maximalen Sexualdimorphismus,

3. Form und Funktion sind für die Art spezifisch und unentbehrlich und schließlich,

4. Er kommt bereits während der letzten Jugendstadien zur Ausbildung.'

Die Literaturangaben über den Enp. P4 ô wurden also sorgfältig ausgewertet, morphologische Vergleichstabellen angefertigt und schließlich Formreihen aufgestellt, in die nur Arten aus jeweils gleicher biogeographischer Region Auf- 


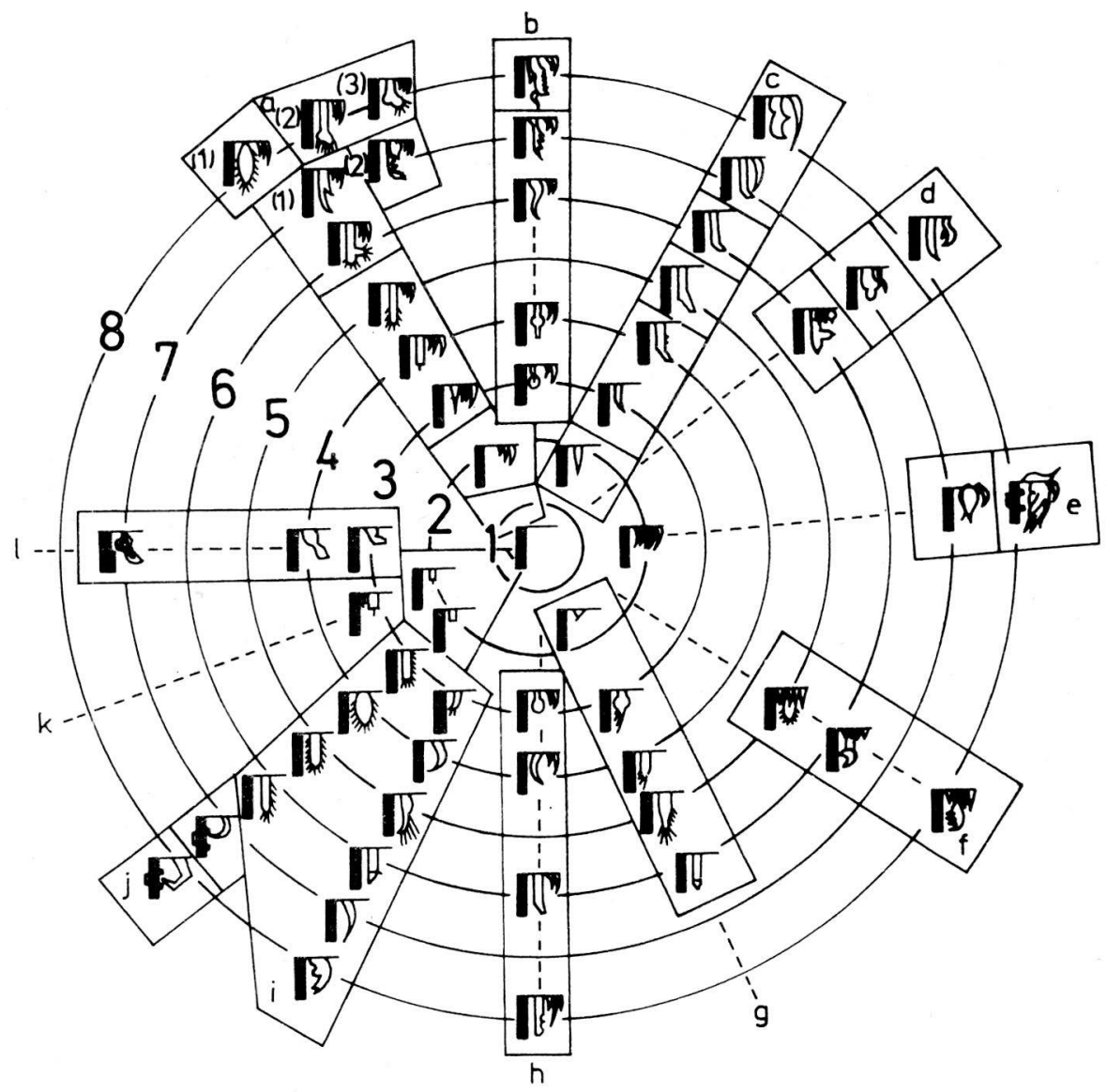

Abb. 1. Schalenmodell der Enp. $\mathbf{P}_{4} \hat{\delta}$-Trendreihen nach Jakobi, 1972a, abgeändert durch Umrandung der den einzelnen Gattungen zugeordneten Typen (1 Kasten = 1 Gattung).

nahme fanden. Das Aufstellen solcher Reihen wurde dadurch erleichtert, daß die verschiedenen Enp. P4 $\delta$ graphisch 'durch Weglassen streng arteigener Charakteristika (1972a, p. 129)' auf ihren Grundgehalt reduziert wurden. Zur bestmöglichen Wiedergabe der Reihen, die Jakobi als Trendreihen bezeichnet, wurde - 'in Analogie zum Atommodell (1972a, p. 132)' - eine Schalenform gewählt. Auf 8 Schalen (Abb. 1) sind dabei die abstrahierten Enp. P4 $\hat{\delta}$ - Typen so angeordnet, daß die relativ einfach gebauten, plesiomorphen Typen, wie Jakobi sie nennt, eine Mittellage einnehmen, also die Schalen 4-6 besetzen. Innerhalb jeder Reihe sollen nun 2 entgegengesetzte Tendenzen zu beobachten sein. Einerseits werden die Endopoditen noch weiter vereinfacht bis hin zur 
totalen Reduktion, andererseits läßt ihr Bau eine zunehmende Differenzierung erkennen. Die Etappen des Reduktionsprozesses sind auf den inneren Schalen (3-1), die des Differenzierungsprozesses auf den äußeren (7-8) wiedergegeben. Genau genommen kann bei einer solchen Anordnung nicht mehr von einer Trendreihe gesprochen werden, denn ein Trend kann schwerlich in zwei unterschiedliche Richtungen laufen.

Welche Kriterien außer geographischem Vorkommen über die Einordnung eines Typs in eine bestimmte 'Trendreihe' entscheiden, und wie die Schalenposition in den noch unvollständigen, von Jakobi als 'ungesättigt' bezeichneten Reihen ermittelt wird, erfahren wir nicht. Es wird nur versichert: 'Die Trendeinreihung macht dem geübten Auge keine Schwierigkeiten (1972a, p. 134)'.

Die Zahl der Trendreihen ist beliebig und hängt davon ab, wie weit die Erforschung der Parastenocarididenfauna einer biogeographischen Region gediehen ist. Im Augenblick vermag Jakobi insgesamt 12 solcher 'Trendreihen' zu erkennen: 4 für die holarktische, 1 für die orientalische, 3 für die äthiopische und 4 für die neotropische Region.

Was aber soll das Schalenmodell nun überhaupt und worin liegt sein Nutzen? Wenn ich Jakobi richtig verstanden habe, hauptsächlich wohl in zweierlei:

1. soll es möglich sein, aus ihm eine Einteilung der Familie in Gattungen abzuleiten und

2. soll es geeignet sein, weitreichende biogeographische Einsichten zu eröffnen. Ich muß jedoch gestehen, daß ich beides nicht nachzuvollziehen vermag.

Wenn eine wichtige Voraussetzung für die Aufstellung einer 'Trendreihe' ist, daß sie nur Arten mit Vorkommen in gleicher biogeographischer Region umfaßt, welch anderen Schluß soll man dann aus ihr ziehen können, als daß afrikanische Arten in Afrika und südamerikanische in Südamerika vorkommen? Kontinentübergreifende Verwandtschaftsbeziehungen werden ja von vornherein ausgeschlossen.

Was das Schalenmodell mit der Einteilung der Familie in 26 (davon 24 neue) Gattungen zu tun hat, die Jakobi anschließend auf 9 Seiten vorlegt, ist ebenfalls undurchsichtig. Von den 147 bis Anfang 1970 (als sich Jakobi zu seiner Revision entschloß) bekannten Arten werden 100 den einzelnen Gattungen zugeordnet, 7 werden nicht berücksichtigt, weil von ihnen nur $q q$ bekannt sind, 5 weitere werden zwar am Rande erwähnt, doch wird offengelassen, zu welcher Gattung sie gehören, die restlichen 35 werden überhaupt nicht genannt.

Vergleicht man Schalenmodell und Gattungseinteilung, so stellt man verwundert fest, daß eine Gattung so ziemlich jede Zahl von Enp. P4 ô - Typen umfassen kann. Mal schließt sie alle Typen einer Reihe von den einfachsten bis hin zu den angeblich höchstdifferenzierten ein, mal nur letztere oder andere allein. Welches die Gründe dafür sind, bleibt unerwähnt. Hatte man vorher noch vermutet, daß jede 'Trendreihe' eine natürliche Verwandtschaftsgruppe darstellen soll, so stellt sich jetzt heraus, daß eine Gattung sogar Typen von 2 verschiedenen 'Trendreihen' in sich vereinigen kann. Zwar wird zugegeben, daß die betreffende Gattung, die statt der üblichen 1-4 insgesamt 24 Arten 
umfaßt, in Zukunft vermutlich noch weiterer Unterteilung bedarf, doch scheinen keine grundsätzlichen Bedenken zu bestehen.
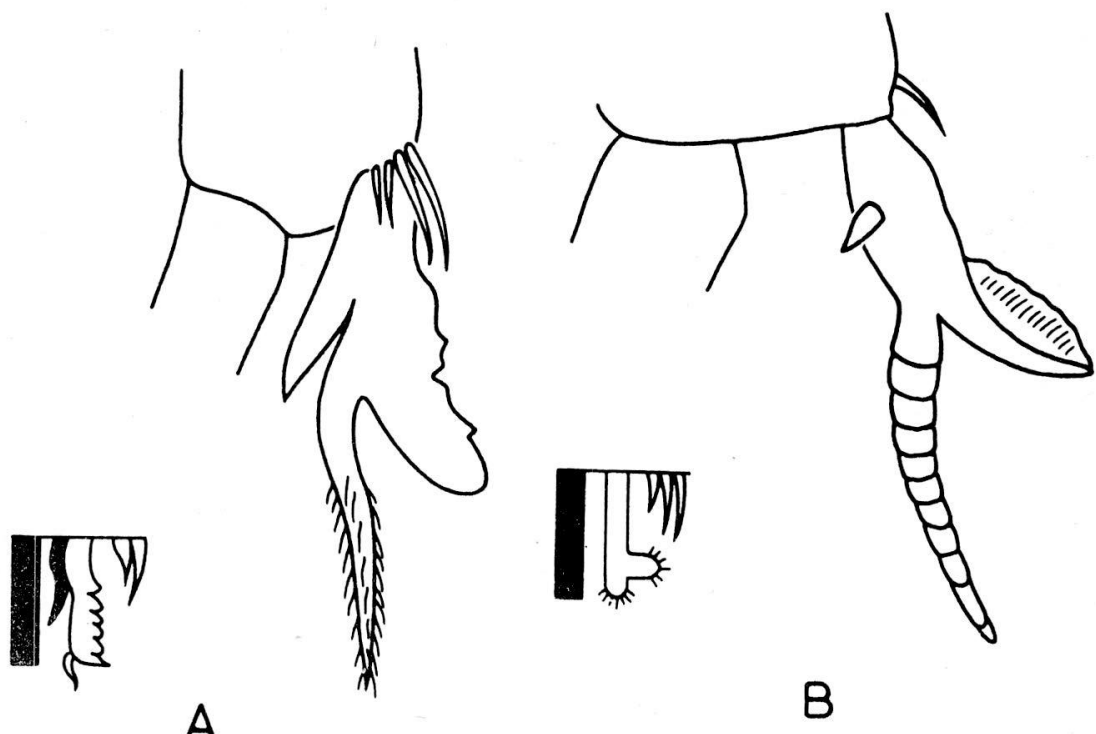

$A b b$. 2. Endopoditen der 4. Peraeopoden $\hat{\delta}$ und deren Typisierung durch Jakobi, 1972a. A - von Parastenocaris phreatica Chappuis, 1936; B - Parastenocaris acherusia Noodt, 1955.

Weder Schalenposition also, noch ganze 'Trendreihen' scheinen gattungsdiagnostischen Wert zu haben, und selbst auf die spezifische Struktur des Endopoditen scheint das nicht zuzutreffen. Sieht man sich die Originalabbildungen der Enp. P4 $\hat{O}$ von Parastenocaris phreatica Chappuis, 1936 und $P$. acherusia Noodt, 1955 an (Abb. 2), so hat man den Eindruck, daß sie sehr ähnlich seien, und wäre der Bau des Enp. P4 $\delta$ für die Ġattungseinteilung ausschlaggebend, so würde man vermuten, daß beide einer Gattung angehören. Das ist aber nicht der Fall, und beide Endopoditen figurieren auch im Modell als gänzlich unterschiedliche Typen, was Position und 'Trendreihe' anbetrifft.

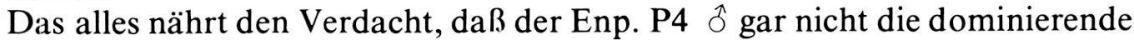
Rolle bei der Gattungseinteilung gespielt hat, vielmehr sind unterschwellig andere Merkmale mit herangezogen worden. Es bleibt unklar, welche Merkmalskombination es nun ist, die eine Gattung eindeutig charakterisiert. Folgende Einleitung zur Gattungsdiagnose von Fontinalicaris gen. n. illustriert diese Unklarheit: 'Dieses Genus reiht sich natürlicherweise an das vorhergehende, sodass natürlicherweise keine scharfe Trennung besteht (1972a, p. 139)'.

Wer Gattungen aufstellt, muß ihre Grenzen sichtbar machen und gleichzeitig 
damit den Nachweis führen, daß alle Arten, die er in ihnen vereinigt, gemeinsamen Ursprungs, d.h. eine monophyletische Gruppe sind. Jakobi macht auch nicht ansatzweise den Versuch dazu. Sein Schalenmodell kann nicht als solcher angesehen werden. Mit seinen Anspielungen auf das Atommodell und das Periodische System der Elemente gehört es vielmehr in die Nachbarschaft der Versuche Bachmetjevs (1903-1904), ein Periodisches System der Schmetterlinge aufzustellen (zitiert nach Hennig, 1950, p. 22).

Will man den tatsächlichen Verwandtschaftsbeziehungen in der Familie Parastenocarididae auf die Spur kommen, wird man neu beginnen müssen Eine isolierte Betrachtung eines Einzelmerkmals und seine Reihung nach Ähnlichkeiten führt dabei nicht weiter. Vielmehr wird der Weg zu beschreiten sein, den Jakobi abschließend selber erkennt: 'Um aber eine traditionelle, mehrere Genuscharakteristika berücksichtigende Diagnose geben zu können, wäre es notwendig, sämtliche bisher bekannten Parastenocarididen zu überarbeiten, um die entsprechenden Eigenschaftsbeziehungen abzugrenzen (1972a, p. 135)'. Wenn das bisher keiner unternommen hat, so liegt dies daran, daß das Typenmaterial sehr vieler Arten nicht mehr zur Verfügung steht. Daß Jakobi mehrere dieser verschollenen Arten jetzt zu Typusarten seiner Gattungen gemacht hat, kommt als weitere Belastung hinzu.

\section{BATHYNELLIDAE}

Der Besprechung der Bemühungen Serbans, die Familie Bathynellidae in den Griff zu bekommen, muß vorausgeschickt werden, daß er mit seiner Arbeit insofern neue Maßstäbe gesetzt hat, als er durch seine eingehenden morphologischen Analysen des Thorakopoden VIII $\hat{o}$ (Th. VIII $\hat{\delta}$ ) einen bedeutsamen Merkmalskomplex erschlossen hat, dem vorher gerade wegen seiner Komplexität stets ausgewichen worden war.

Was die Gliederung der Familie selbst anbetrifft, so leidet sie, wie ich glaube, unter zwei Nachteilen:

1. der frühzeitigen Festlegung auf den Th. VIII $\hat{\delta}$ als 'Hauptmerkmal', die schon sehr bald dazu führt, daß Zusatzhypothe:sen erforderlich werden,

2. dem ausschnitthaften Vorgehen bei der Bearbeitung der Familie.

Da frühere Beschreibungen in wichtigen Punkten zu oberflächlich oder unvollständig sind und Typenmaterial offenbar schwer zu beschaffen ist, arbeitet sich Serban Schritt für Schritt nur mit dem ihm zur Verfügung stehenden Eigenmaterial voran. Auf diesem Wege hat er bisher selbst oder in Zusammenarbeit mit anderen Autoren 16 neue Arten beschrieben und 3 nachuntersucht, die er auf 6 neue Gattungen und 2 schon bekannte verteilt, sowie 3 Unterfamilien als erster abgegrenzt.

Dreh- und Angelpunkt dabei ist wie gesagt der Th. VIII $\hat{o}$. Dabei handelt es sich um ein vermutlich ehemaliges Schreitbein, das teils auf neotenischem Wege durch von Fall zu Fall unterschiedlich wirksame Blockade der Ausdifferenzierung von Exo- und Endopodit, teils durch zusätzliche Spezialisierungs- 
prozesse, wie Ausbildung eines Penisanhangs, Verformung und unterschiedliche Artikulation der unfertigen Spaltbeinäste, zu einem komplizierten Organ im Dienste der Fortpflanzung wurde. Von der komplexen Struktur dieses Organs hat sich Serban, wie ich finde, dann zu sehr beeindrucken lassen, so daß er sogar so weit ging, den Th. VIII $\hat{\delta}$ ausdrücklich zum 'Hauptmerkmal' (caractère primordial) zu erklären (Serban, 1971, 1973a), und ihn allen übrigen, pauschal als 'sekundäre (secondaire) oder untergeordnete (subordonné)' bezeichneten Merkmalen gegenüberzustellen. Seine strukturelle Plastizität schien Serban getreulicher als alle Veränderungen an den übrigen Körperanhängen die evolutorischen Prozesse wiederzuspiegeln, die sich innerhalb der Bathynellacea abgespielt und zur Entstehung der Formenvielfalt geführt hatten. Mit dieser Zweiteilung in 'Haupt- und Nebenmerkmale' wird aber eine künstliche Wertung vorgenommen, die nur nachvollziehbar ist, wenn man das Tier losgelöst von seiner Biologie und seinen Umweltbeziehungen betrachtet. Die pauschale Bezeichnung 'sekundäre Merkmale' für alle Körperanhänge (von $\hat{\delta}$ und $q$ und wohl auch Jugendstadien!) außer dem Th. VIII ồ hätten allenfalls einen Sinn, wenn alle die gleiche Funktion ausübten.

Bei der Bearbeitung osteuropäischen Materials kam Serban mit diesem Konzept dennoch ganz gut voran, und auch die Nachuntersuchung von $\mathrm{Au}$ strobathynella patagonica aus Südamerika (Delamare Deboutteville \&Serban, 1973) schien das Konzept zu bestätigen. In den 'sekundären Merkmalen' gleicht diese Art so sehr den europäischen Formen, daß Noodt (1965) sie noch für kongenerisch mit diesen gehalten hatte. Erst die Analyse des 'Hauptmerkmals' bringt letzte Klarheit und läßt keinen Zweifel an der Eigenständigkeit der südamerikanischen Art. Im Bau des Th. VIII $\hat{\sigma}$ weicht sie sogar so von den übrigen Bathynellidae ab, indem sie Anklänge an die Verhältnisse bei den Parabathynellidae zeigt, daß, wie Serban (1973a, p. 212) selbst ausführt, 'en absence des traits secondaires il nous aurait été impossible de soutenir l'appartenance de l'espèce à la famille des Bathynellidae'. Hätte man daraus nicht auch eine andere Lehre ziehen können? Denn was ist eine Unterscheidung in Haupt- und Nebenmerkmale wert, wenn die Nebenmerkmale eine so überragende Bedeutung bekommen können?

Erste wirkliche Schwierigkeiten mit seinem Konzept bekam Serban bei der Untersuchung südwesteuropäischer Formen (Serban, Coineau, Delamare Deboutteville, 1972), die im Bau des Th. VIII ô erstaunlich einheitlich, in den "sekundären Merkmalen" aber bemerkenswert unterschiedlich sind. Nach dem Th. VIII $\hat{o} \mathrm{zu}$ urteilen, gehörten alle in eine Gattung, doch schien Serban et al. die Vielfalt der “sekundären Merkmale" das nicht zuzulassen. Also gliedern sie die 8 Arten mit Hilfe dieser Merkmale in Gattungen und vereinigen sie wagen des einheitlichen Baus des Th. VIII $\hat{s}$ in einer eigenen Unterfamilie.

Abgesichert und gerechtfertigt wird dieses Vorgehen später (Serban, 1973a) durch die Zusatzhypothese, daß offenbar nicht alle Bathynellidae vergleichbar sind, was die Modalitäten ihrer Evolution betrifft. Das heißt, im einen Fall kann mal die Entfaltung des "Hauptmerkmals", dafür im anderen mehr die der "sekundären Merkmale" begünstigt werden. Das Ergebnis ist in jedem Fall, was Serban “die kompensatorische Vielfalt der sekundären Merkmale im Ver- 
hältnis zum Hauptmerkmal" (diversification compensatrice des caractères secondaires par rapport au caractère primordial) nennt. Auf eine kurze Formel gebracht, heißt das: Monotonie im Bau des "Hauptmerkmals" wird durch Vielfalt im Bau der "sekundären Merkmale" kompensiert und umgekehrt.

Es ist klar, daß diese Vorstellungen wieder nur losgelöst von jeglichem biologischen Hintergrund nachvollziehbar sind. Erstens erscheint es mir unwahrscheinlich, daß sich die Modalitäten der Evolution ausgerechnet in einem so konstante Milieubedingungen bietenden und konkurrenzarmen Lebensraum wie dem Mesopsammal ändern sollten. Dazu bedürfte es schon variabler Bedingungen oder gar eines Lebensraumwechsels. Zweitens ist "Strukturelle Kompensation" zwischen Organen unterschiedlicher Funktion ganz undenkbar. Welche Notwendigkeit sollte denn bewirken, daß eine Gruppe zum Ausgleich für mangelnde Vielfalt etwa im Bau der Kopulationsorgane plötzlich, sagen wir, komplizierte Antennen, also Tastorgane, entwickelte? Biologisch sinnvoll wäre das doch nur, wenn die Antennen sekundär auch eine Rolle bei der Kopulation übernähmen und etwa durch Abwandlung zu einem Greiforgan komplizierte Hakenbildungen am eigentlichen Kopulationsorgan selbst überflüssig machten. Die Unterscheidung in Haupt- und Nebenmerkmale führt also zu einem Schematismus, der einfach unbiologisch ist.

Vollends unhaltbar wird er, wenn Formen auftauchen, die eine Zwischenstellung zwischen den auf Grund des "Hauptmerkmals" abgegrenzten Großgruppen (Unterfamilien) einnehmen. Die Gattung Sardobathynella ist ein solcher Fall (“...présentant des tendances évolutives qui zigzaguent entre les deux sous-familles...”! 1973b, p. 106), und sie nötigte Serban zu dem Bekenntnis (1973b, p. 103): "Il nous faut reconnaître que les informations assez relatives empêchent l'identification des potentialités réelles du processus de diversification dans le groupe et que celui-ci, trop inégal dans les diverses lignées, est déroutant en ce qui concerne la valeur taxonomique qu'on doit accorder aux caractères". Aus diesem Bekenntnis geht hervor, daß klare Vorstellungen darüber noch fehlen, wie die einzelnen Merkmale zu bewerten sind! Der Ausschnitt gesicherter Erkenntnis ist also noch zu klein, um einen ausreichenden Überblick über die Abwandlungsmöglichkeiten der Körperanhänge zu gestatten, ohne den eine verläßliche Taxierung der Bewertungsmaßstäbe jedoch unmöglich ist.

In diesem Zusammenhang muß auf ein Phänomen hingewiesen werden, das bisher kaum Beachtung gefunden hat. Jakobi $(1954$, p. 27) macht darauf aufmerksam, daß bei den Bathynellidae zwischen erwachsenen und geschlechtsreifen Tieren zu unterscheiden ist: "Mit der vollständigen Ausbildung der Extremitäten (= erwachsen) ist aber keineswegs ein Abschluß der Häutungen verbunden." Wichtig ist nun, daß die Erwachsenenhäutungen noch strukturelle Veränderungen bringen können. So berichtet Jakobi selbst von einem Weibchen mit einer gekrümmten Querborste an der Basis und einem rasierpinselförmigen Gebilde am Grundglied des Protopoditen des Th. VIII und schreibt (1954, p. 40): "Erwähnen muß ich noch, daß diese beiden Gebilde beim eben erwachsenen Weibchen noch nicht zu finden sind, sondern erst nach der 1. oder 2. Erwachsenenhäutung hinzukommen." 
Ich selbst hatte kürzlich Gelegenheit, Bathynellen aus der Umgebung von Karlsruhe zu untersuchen, wobei in einer Probe größere und kleinere Männchen vorkamen, die im Bau des Th. VIII ô nicht voneinander abwichen. Die größeren hatten aber Mandibelpalpen mit unterschiedlich gebauten Endklauen, die bei den kleineren Männchen und bei den Weibchen derselben Probe gleichgestaltet waren. Es ist zu vermuten, daß dieser Sexualdimorphismus im Bau der Mandibelpalpen, der erstmalig für die Gattungen Pseudobathynella und Sardobathynella beschrieben worden ist, auch erst ein Produkt der Erwachsenenhäutungen ist. Damit stellt sich aber die Frage, basieren alle Beschreibungen von Bathynellidae wirklich auf voll ausdifferenzierten Exemplaren?

Dies und die noch bestehende Unsicherheit bei der Einschätzung der Wertigkeit der Merkmale in der Familie Bathynellidae ließen es ratsam erscheinen, von einer weiteren Untergliederung der Familie in Gattungen vorerst abzusehen und sich mit der Andeutung von Artengruppen zu begnügen, bis Nachuntersuchungen schon bekannter und zukünftige Beschreibungen neuer Arten ausreichend Material geliefert haben, damit statt einer portionsweisen Gattungseinteilung mit parallellaufender Theorienbildung eine geschlossene Revision aus einem Guß ins Auge gefaßt werden kann.

\section{PARABATHYNELLIDAE}

Die Gattungsgliederung der Familie Parabathynellidae (Schminke, 1973) geht zunächst von den 3 Merkmalskomplexen Mundwerkzeuge, Th. VIII $\hat{\delta}$ und Antenne II (A. II) aus, die als "Orientierungsmerkmale" bezeichnet werden können. Alle Arten einer Gattung müssen im Bau dieser 3 Merkmalskomplexe $\mathrm{im}$ wesentlichen übereinstimmen. Das heißt aber nicht, daß sie deshalb den übrigen Merkmalen übergeordnet wären, so daß sie in jedem Fall bei der Gattungsgliederung den Ausschlag gäben, gleichgültig ob die übrigen Merkmale harmonieren oder nicht. Ihre Heranziehung als "Orientierungsmerkmale" geschieht aus folgenden Gründen:

1. Alle drei haben eine jeweils andere Funktion (A. II: Seitentaster, Mundwerkzeuge: Nahrungsaufnahme, Th. VIII ô : Kopulation), so daß angenommen werden kann, daß sie sich unabhängig voneinander entwickeln, und nicht etwa wie die Mundwerkzeuge gemeinsam im Hinblick auf eine einheitliche Funktion. Die Mundwerkzeuge können deshalb auch nicht jedes für sich als selbständiges Merkmal gewertet werden, sondern nur zusammen als einheitlicher Merkmalskomplex.

2. Die Mundwerkzeuge und der Th. VIII ô sind sehr komplex gebaute Organe - die kompliziertesten des Parabathynellenkörpers überhaupt - und ihre Abwandlungen sind auch nicht durch bloße Reduktionsvorgänge zu erklären, sondern als spezifische Umbildungen mit komplizierendem Charakter. Es ist daher wahrscheinlich, daß Veränderungen an ihnen eher Anzeichen eines einmaligen Vorganges sind, der eine durch diese Veränderungen gekennzeichnete Gruppe als natürliche monophyletischer Abstammung 
ausweist, als etwa Veränderungen an Merkmalen wie z.B. der A. II, die auf Reduktionen gründen, und bei denen man nie weiß, ob sie monophyletisch oder mehrmals parallel entstanden sind.

3. Es hat sich gezeigt, daß Artengruppen, die durch Übereinstimmungen in diesen 3 Merkmalskomplexen gekennzeichnet sind, auch noch weitere Merkmale (z.B. komplexes Antennalorgan, Bau der Thorakopodenexopoditen, des Analoperculums usw.) gemeinsam haben, die allerdings nicht von Gruppe zu Gruppe dieselben sein müssen.

Für die Grenzziehung zwischen Gattungen ist es nun wichtig, alle verfügbaren Merkmale zu bewerten. In der Regel wird man wegen ihres hohen Informationsgehaltes mit den 3 "Orientierungsmerkmalen" für die Grenzziehung auskommen. Doch es gibt durchaus Fälle, wo sie allein nicht genügen. Ein solcher Fall wird weiter unten zu besprechen sein. Es ist nun keineswegs nötig, daß sich verschiedene Gattungen stets in allen 3 "Orientierungsmerkmalen" gleichzeitig voneinander unterscheiden. Sie können vielmehr in einem oder auch zweien Übereinstimmungen aufweisen. Doch ist zu beachten, daß die übereinstimmenden Merkmalskomplexe in ihrer Ausprägung immer einen Zustand repräsentieren müssen, der dem Ausgangszustand der gemeinsamen Stammart sehr nahe steht, und daß Veränderungen speziell der A. II allein wegen ihres Zustandekommens auf Grund von Reduktionsvorgängen in keinem Fall ausreichen, um eine Gattung zu begründen.

In der Regel unterscheiden sich die Gattungen in mehr als nur einem der 3 "Orientierungsmerkmale", es kann im Extremfall aber auch 2 Gattungen geben, die sich nur im Bau des Th. VIII ồ unterscheiden, im Bau der A. II und der Mundwerkzeuge aber ähnlich sind. In diesem Fall ist eine Trennung beider nur dann statthaft, wenn A. II und Mundwerkzeuge bei beiden Gattungen auf einem dem Ausgangszustand bei der gemeinsamen Stammart ähnlichen Zustand verharren, während die Veränderungen am Th. VIII $\hat{o} \mathrm{mehr}$ als eine Modifizierung des Ausgangstyps, also so einschneidend sind, daß ein gänzlich neuer Typ entsteht. Das heißt, nicht nur die durch diesen neuen Typ gekennzeichneten Arten müssen durch ihn als monophyletische Gruppe ausgewiesen werden, sondern auch die verbleibenden Restarten durch den Typ ihres Th. VIII $\hat{o}$.

Auf der anderen Seite ist von 2 Gattungen, die nur im Bau der Mundwerkzeuge differieren, zu fordern, daß bei ihnen Th. VIII ô und A. II dem Ausgangszustand bei der gemeinsamen Stammart relativ ähnlich bleiben, die Veränderung des Komplexes "Mundwerkzeuge" dagegen so beschaffen ist, daß daraus auf die Einmaligkeit dieser Entwicklung geschlossen werden kann. Da aber wie gesagt Arten, sofern sie in den 3 Merkmalskomplexen A. II, Mundwerkzeuge und Th. VIII $\hat{o}$ übereinstimmen, stets noch in weiteren Merkmalen identisch sind, können in Zweifelsfällen auch diese zur Beurteilung herangezogen werden, und von einem solchen Fall soll jetzt die Rede sein.

In einer Probe aus Südafrika sind 2 Arten aufgetaucht (Beschreibungen vgl. Anhang), die im Bau des Th. VIII ô ähnlich sind (Tafel 3 und 5), sich in dem der A. II (Tafel 2,1 und 4,2) und der Mundwerkzeuge (Tafel 1, 2-5 und 4, 3-6) aber unterscheiden. Doch muß zugegeben werden, daß die Unterschiede der 
Mundwerkzeuge nicht so erheblich sind wie sonst, wenn sie für die Trennung zweier Gattungen den Ausschlag geben. Trotzdem werden beide Arten als Vertreter selbständiger Gattungen, Afrobathynella gen. n. und Nunubathynella gen. n., angesehen. Die Gründe dafür sind:

1. Die Thorakopoden VIII $\hat{\delta}$ beider Gattungen sind relativ plesiomorph, und ihre Übereinstimmung wiegt daher nicht so schwer bei der Beurteilung der Gattungszugehörigkeit.

2. Schwerer wiegen dagegen die verglichen mit Afrobathynella gen. n. apomorphe Abwandlung der Mundwerkzeuge der Gattung Nunubathynella gen. n. und die damit korrelierte apomorphe Form ihrer A. II.

3. Nunubathynella gen. n. weist zusätzliche apomorphe Merkmale auf, die, würde sie zusammen mit Afrobathynella gen. n. einer einzigen Gattung angehören, das Maß der Unterschiede weit überstiegen, das bisher zwischen Arten gleicher Gattung zu verzeichnen ist. Nunubathynella gen. n. hat ein komplexes Antennalorgan, eingliedrige Thorakopodenexopoditen, eine Dornenreihe am Uropodensympoditen, deren vorletzter Dorn vergrößert ist, und einen Uropodenendopoditen in Stilettform. Afrobathynella gen. n. ist in allen diesen Merkmalen urtümlicher.

Will man nun überprüfen, ob die Gattungsgliederung einer Familie nach morphologischen Kriterien wirklich zu einer natürlichen Gruppierung geführt hat, so kann man sich nach ökologischen, zoogeographischen oder ethologischen Anhaltspunkten umsehen. Umgekehrt allerdings geht es nicht. Morphologische Kriterien können durch anderweitige Anhaltspunkte nicht ersetzt werden.

In vielen Fällen ist nachgewiesen worden (Mayr, 1969, Illies, 1970), daß einer Gattung eine ganz bestimmte Nische oder adaptive Zone entspricht. Im vorliegenden Fall ließen sich dafür aus der Koexistenz verschiedener Arten gewisse Anhaltspunkte ableiten. Nach der Revision der Familie fiel auf (Schminke, 1973a), daß alle Arten, die gemeinsam in derselben Probe angetroffen worden waren, zu verschiedenen Gattungen gehören. Morphologische Vergleiche ergaben, daß unterschiedliche Körpergröße und verschiedener Bau der Mundwerkzeuge vermutlich dafür verantwortlich sind, ob 2 Arten nebeneinander bestehen können. Tiere mit ähnlichen Mundwerkzeugen können nur koexistieren, wenn sie sich deutlich in der Körpergröße unterscheiden, so daß sie dadurch gegenseitige Konkurrenz vermeiden können, dāß sie in Mischsubstraten mit unterschiedlich großen Lückenräumen diejenigen besiedeln, die ihrer Größe angemessen sind. Tiere von gleicher Körpergröße dagegen können nur koexistieren, wenn sie sich auf andere Weise eine Nische erschlossen haben, und der unterschiedliche Bau der Mundwerkzeuge bei ihnen deutet an, worin diese Nische bestehen könnte: in unterschiedlichen Arten der Nahrungsbeschaffung bzw. ihrer Bearbeitung.

Was zoogeographische Hinweise anbetrifft, so hat sich herausgestellt, daß die Gattungen der Familie Parabathynellidae mit Ausnahme von Hexabathynella, die sekundär in die Nähe des Meeres vorgedrungen ist (Schminke, 1972), alle ein einheitliches Areal besiedeln, das, sofern es heute durch getrennte Landmassen auseinandergerissen ist, durch paläogeographische Rekonstruktionen als ehemals zusammenhängend erkannt werden kann. 
Die Gliederung der Familie Parabathynellidae in 19 Gattungen hat also sowohl ökologisch als auch zoogeographisch eine gewisse Bestätigung erfahren. Zukünftige Untersuchungen werden verstärkt den Verwandtschaftsbeziehungen zwischen den Gattungen gewidmet sein. Neue Anhaltspunkte dafür dürften von einer vertieften Analyse des Th. VIII $\delta$, so wie Serban sie initiiert hat, zu erwarten sein.

\section{SCHLUßBEMERKUNGEN}

Überdenkt man abschließend noch einmal die drei geschilderten Revisionsversuche, so verdienen unabhängig von den jeweiligen Besonderheiten der einzelnen Fälle folgende Punkte noch einmal hervorgehoben zu werden:

1. Ein biologisches System klassifiziert Lebewesen und nicht Merkmale. Jedes Lebewesen ist durch vielfältige Bindungen in eine Umwelt eingepaßt, der gegenüber es sich zu behaupten hat. Wer sich dieser Beziehungen auch bei der Arbeit mit konserviertem Material stets bewußt bleibt und nicht vergißt, Merkmale auch in Zusammenhang mit Selektion und Anpassung zu sehen, wird nie ein einziges Merkmal allein, sondern alle gleichzeitig im Auge haben.

2. Ein System ist der Vielfalt des Lebens nicht vorgeordnet, sondern kann nur aus ihr abgeleitet werden. Es ist daher sinnlos, diese Vielfalt in ein vorgegebenes Schema pressen zu wollen. Lebewesen sind eben keine Elemente. Sie verdanken ihre Entstehung einem langen historischen Prozeß, und im Natürlichen System werden Organismen deshalb weniger nach dem Grad ihrer Ähnlichkeit geordnet, als vielmehr nach dem ihrer Verwandtschaft, des $\mathrm{Zu}$ rückliegens ihrer jeweils gemeinsamen Abstammung.

3. Verwandtschaft kann nur durch Bewertung von Merkmalen ermittelt werden. Wer Lebewesen rein nach Ähnlichkeiten ohne deren Bewertung zu ordnen versucht, verfährt nach den scholastischen Methoden der Bestimmungsschlüssel. Der Nachweis von Verwandtschaft kann nur durch das Auffinden von Synapomorphien erbracht werden (Hennig, 1950; Schlee, 1971). Alleein das gemeinsame Vorhandensein eines abgeleiteten Merkmals deutet auf engste Verwandtschaft hin, wobei allerdings gilt, daß dieses Merkmal nicht als mehrfach unabhängig entstanden gedacht werden kann. Konvergenz ist umso unwahrscheinlicher, je strukturreicher ein sich verändernder Merkmalskomplex ist. Insofern ist der Informationsgehalt aller Merkmale nicht gleich. Es gibt solche, die wegen ihres komplexen Baus höherwertig als andere sein können. Man hüte sich aber, sie deshalb voreilig zum "Hauptmerkmal" zu erheben. Prinzipielle Umbauten an anderen Strukturen oder korreliertes Auftreten anderer, funktionell unabhängiger Komplexe können ihren vermeintlich universellen Indikatorwert schnell fragwürdig machen.

4. Synapomorphien charakterisieren eine Gruppe als monophyletische Einheit, die auf eine gemeinsame Stammart zurückgeführt werden kann. Zur Aufstellung einer Gattung gehört, daß die Monophylie ihrer Arten eingehend begründet und nicht nur behauptet wird. Es gehört auch dazu, daß die 
Monophylie des verbleibenden Restes der Arten, die nicht in diese Gattung aufgenommen werden, ebenfalls unter Beweis gestellt wird.

5. Es ist deshalb unmöglich, Unterfamilien zu definieren oder Gattungen aufzustellen, wenn man nicht einen hinreichenden Überblick über die ganze Familie hat. Wer mit der Bearbeitung eines Sektors beginnt und versucht, die dabei gewonnenen Einsichten zu verallgemeinern, wird bei einer Ausweitung seiner Arbeiten durch das überraschende Auftreten von Neuheiten überrollt werden, die im voraus nicht kalkulierbar waren und die die bis dahin geleistete Arbeit selbst revisionsbedürftig machen können.

6. Wer eine größere Gruppe überblickt, wird erkennen, daß ein einziges Merkmal nicht ausreicht, die Vielfalt zu ordnen, und schon gar nicht, die Monophylie der erkannten Gruppierungen in jedem Fall nachzuweisen. Er wird sich sogar der Beschränkung bewußt werden, die in der alleinigen Betrachtung der externen Adultmorphologie liegt und zusätzliche Merkmale einstweilen außer acht läßt, die sich aus der inneren Anatomie, dem Entwicklungszyklus, der Ökologie, der geographischen Verbreitung usw. ergeben. Die Überzeugung, mit einem Merkmal auszukommen, kann nur hegen, wer sich zunächst nur auf einen Ausschnitt der Gesamtheit konzentriert. Sobald er darüber hinausgeht, wird er sich gezwungen sehen, entweder weitere Merkmale einzubeziehen oder an seinem "Hauptmerkmal" immer größere Feinheiten zu berücksichtigen, d.h. mehr die Unterschiede als die Gemeinsamkeiten zu beachten. Dabei sind Gattungen, worauf Inger (1958) mit Recht hingewiesen hat, im Gegensatz zu Arten synthetische Kategorien. Für das Erkennen von Arten gilt es, das Hauptaugenmerk auf Unterschiede zu richten, für das Erkennen von Gattungen aber, den Blick für Gemeinsamkeiten offenzuhalten.

Hin und wieder wird geklagt, daß die systematische Bearbeitung von Grundwasserkrebsen wegen ihres meist einfachen Körperbaus und des Mangels hinreichend komplexer Strukturen so schwierig sei. In Wirklichkeit rührt die Mehrzahl der Schwierigkeiten daher, daß gerade die komplizierten Strukturkomplexe unzureichend beschrieben werden, und der Hauptehrgeiz darauf gerichtet ist, immer neue Taxa aufzustellen, ohne gleichzeitig ihre phylogenetischen Verwandtschaftsbeziehungen aufzuzeigen und im System zum Ausdruck kommen zu lassen. Die systematische Bearbeitung der Grundwasserkrebse ist nicht problematischer als die anderer Gruppen auch, wollte man nur erst einmal die vorhandenen Möglichkeiten, sowohl in deskriptiver als auch methodischer Hinsicht, richtig ausschöpfen.

\section{ANHANG: ARTBESCHREIBUNGEN}

\section{Afrobathynella trimera gen. n., sp. n.}

\section{Gattungsdiagnose}

Thorakopod VIII ô mit dreilappigem Penisanhang und kleiner caudaler Vorwölbung des Grundgliedes; Grundglied außen ohne Abgrenzung in keilförmigen Epipoditen übergehend, der sich 
Tafel 2

Afrobathynella trimera gen. n., sp. n. $\hat{\delta}_{1}$ und $q_{1}$

1. - Antenne II $\hat{\delta}_{1} ; 2$. - Thorakopod I $\hat{\delta}_{1} ; 3$. - Thorakopod III $\hat{\delta}_{1} ; 4$. - Thorakopod VII $\hat{o}_{1} ; 5$. Thorakopod VIII und 1. Pleopod $Q_{1} ; 6$. - Pleotelson $\hat{\delta}_{1}$ (dorsal); 7. - Pleotelson $\hat{\delta}_{1}$ (lateral); $7 \mathrm{a}$. - Furkalorgan.

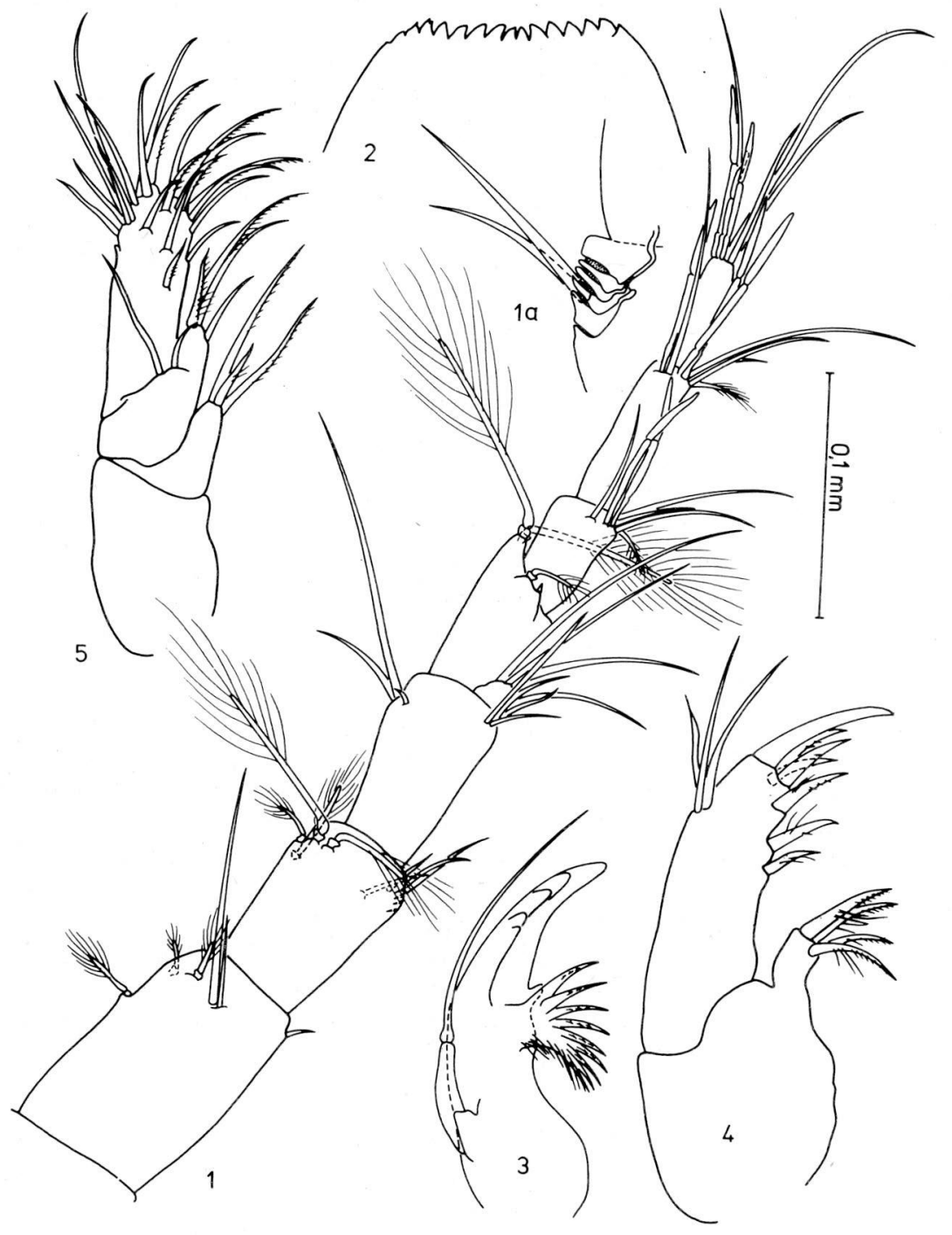

Tafel I

Afrobathynella trimera gen. n., sp. n. Ô.

1. - Antenne I; Ia. - Antennalorgan (dorsal); 2. - Labrum; 3. - Mandibel; 4. - Maxille I; 5. Maxille II 


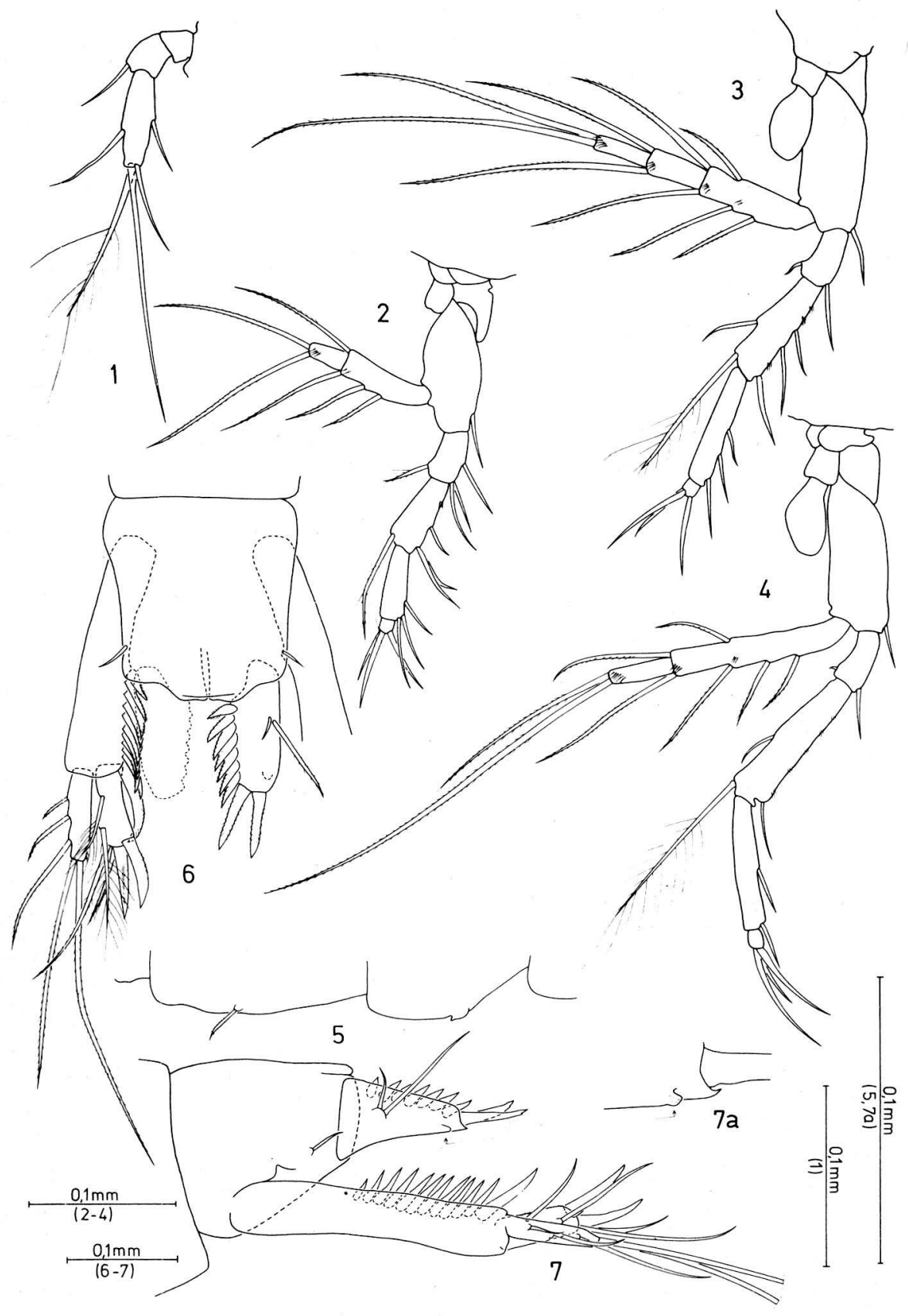




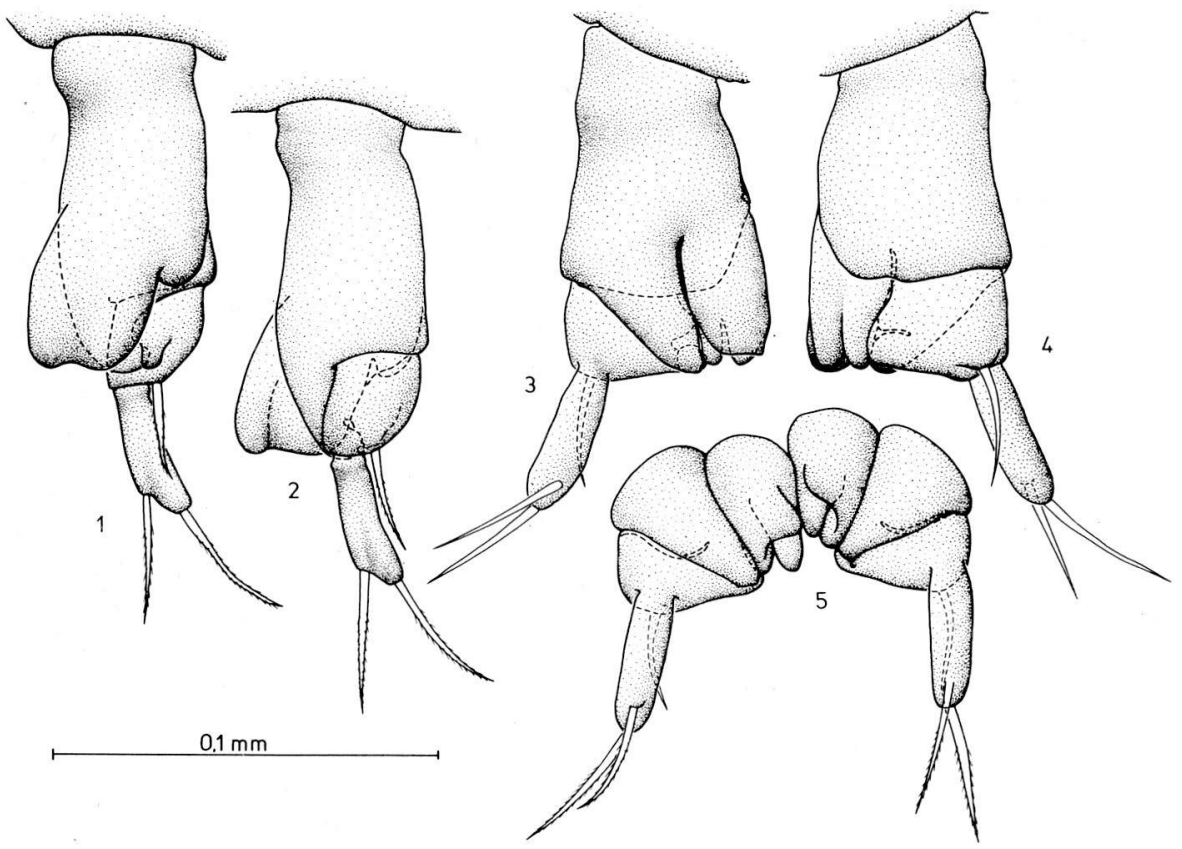

Tafel 3

Afrobathynella trimera gen. n., sp. n. $\hat{\sigma}_{1}$

1. - Rechter Thorakopod VIII (latero-intern); 2. - Linker Thorakopod VIII (latero-extern); 3. Thorakopod VIII (frontal von vorn); 4. - Derselbe (frontal von hinten); 5. - Beide Thorakopoden VIII, distaler Teil (apikal).

nach innen hin zwischen Penisanhang und Basis schiebt. Basis ebenfalls keilförmig mit zum Epipoditen hin umgebogener Spitze; Basis mit einer Borste neben dem Endopoditen. Endopodit gestreckt mit 2 terminalen Borsten. Kein Expodit. Antenne II dreigliedrig, mit 2 kurzen proximalen Gliedern und einem langen distalen Glied; 2. Glied mit einer Innenrandborste, distales Glied mit 3 terminalen Borsten und je einer weiteren Borste auf halber Länge am Innen- und Außenrand. Mundwerkzeuge urtümlich, Maxille II dreigliedrig. Exopoditen der Thorakopoden I-VII zweibis viergliedrig. 1. Pleopodenpaar als kleine Wärzchen mit apikaler Borste vorhanden. Sympodit der Uropoden mit inhomonomem Dornenkamm; Endopodit mit 2 abgesetzten Dornen. Antenne I siebengliedrig, mit Antennalorgan. Furka mit vielen Dornen bewehrt.

Typusart: Afrobathynella trimera gen. n., sp. n.

Material: $\hat{\delta}_{1}$ (Holotyp, als Dauerpräparat auf 7 Objektträgern), $Q_{1}$ (total, als Dauerpräparat).

Probe ZA-83L vom 8.8.1974; Koega River (?), unweit Smitskraal, an der Straße Patensie-Willowmore, Eastern Cape, South Africa. Eine Grabung von $95 \mathrm{~cm}$ Tiefe im mit Steinen durchsetzten Grobkies des weitgehend trockenliegenden Flußbettes (darunter Lehm), 11,00 m vom oberirdischen Lauf des Wassers entfernt; Temperatur: $14^{\circ} \mathrm{C}$, Temperatur des oberirdischen Wassers: $16^{\circ} \mathrm{C}$.

Begleitfauna: Nematoden, Oligochaeten, Cyclopiden, Harpacticiden, Isopoden, Amphipoden, Bathynellidae, Nunubathynella dimera gen. n., sp. n., Leptobathynella spec. 
Beschreibung des Typusexemplars: $\hat{\delta}$ ।

Große Parabathynellidae von 2,70 mm Länge; 15 mal so lang wie die größte Breite; etwas höher als breit; Kopf $40 \%$ länger als breit.

Analoperculum gerade, nicht vorspringend.

Furka 3 mal so lang wie breit, mit 2 größeren Dornen terminal und 7 kleineren am Innenrand; die äußere der dorso-lateralen Borsten 3,5 mal so lang wie die innere; Furkalorgan klein, ventrolateral.

Antenne I siebengliedrig, 30\% länger als der Kopf; Beborstung vgl. Tafel 1,1; Antennalorgan mit 6-7 spaltförmigen Öffnungen, die mit einem "granulierten Material" gefüllt sind; basal geht der Spalt in eine dunkel sich abzeichnende Linie über, die sich nach kurzem, gewundenem Verlauf mit den von den anderen Spalten kommenden Linien vereinigt.

Antenne II dreigliedrig, 80\% kürzer als Antenne I; weitere Einzelheiten vgl. Tafel 2,1.

Labrum mit 10 Hauptzähnen.

Mandibel mit 5 Zähnen am distalen Teil der pars incisiva und einem Borstenlobus mit 9 Klauen. Die Endborste des Palpus ist 3 mal so lang wie das längliche Grundglied. Maxille I vgl. Tafel 1, 4.

Maxille II dreigliedrig; distales und vorletztes Glied verschmolzen, eine Naht noch schwach sichtbar; 2 spaltförmige Öffnungen am Außenrand unterhalb dieser Naht. Borstenformel: 3-6-21 (12-9 bei Berücksichtigung der Naht).

Thorakopoden von Th. I-VII länger werdend; Th. II-VII mit je I Epipoditen; Basis von Th. I-VII mit einer Innenrandborste; Gliederzahl der Exopoditen von Th. I-VII: 2-3-3-3-4-4-3; Zahl der dorsalen Borsten am Exopoditen von Th. I-VII: $1 / 2 / 2-3 / 3 / 3 / 3 / 2$, Zahl der ventralen Borsten: 3/4/4-5/5/5/5/4-5; Endopodit stets viergliedrig, Borstenformeln:

\begin{tabular}{|c|c|c|c|}
\hline Th. I & $3,4+1 / 4+1 / 3+0 / 3(1)$ & Th. & $1+1 / 2+2 / 2,3+0 / 3(1)$ \\
\hline Th. II & $2+1 / 3+2 / 3+0 / 3(1)$ & Th. VI & $1+1 / 2+2 \quad 2+0 / 3(1)$ \\
\hline Th. III & $1+1 / 3+2 / 3+0 / 3(1)$ & Th. VII & $1+1 / 1+2 /$ \\
\hline Th. IV & $1+1 / 2,3+2 / 3+0 / 3(1)$. & & \\
\hline
\end{tabular}

Th. VIII mit gestrecktem Grundglied, das sich innen nach vorn in einen dreilappigen Penisanhang verlängert, dessen schlitzförmige Öffnung cranial vom 3. Lappen abgedeckt wird. Innen caudal neben dem Penisanhang befindet sich eine kleine, rundliche Vorwölbung des Grundgliedes. Außen geht das Grundglied ohne Abgrenzung in den Epipoditen über, der, sich verjüngend, nach innen reicht und sich gleichsam keilförmig zwischen Penisanhang und Basis legt. Basis nach innen ebenfalls spitzer werdend. Die Spitze biegt schließlich um und weist zum Epipoditen hin, so daß in Seitenansicht der Eindruck entsteht, als sei ein kleiner Exopodit vorhanden. Ein solcher fehlt aber, dafür ist der Endopodit auffällig verlängert und trägt terminal 2 Borsten. Auf der Basis hinter dem Endopoditen befindet sich ebenfalls eine kräftige Borste. Beide Th. VIII stoßen in der Mitte mit dem Penisanhang aneinander.

1. Pleopodenpaar in Form zweier Wärzchen mit apikaler Borste vorhanden.

Sympodit des Uropoden 5 mal so lang wie breit mit insgesamt 11-12 Dornen, von denen der distale kräftiger als die übrigen ist. Endopodit $45 \%$ kürzer als Sympodit, mit 2 abgesetzten kräftigen Dornen und außen insgesamt 4 Borsten; Innenrand mit 3 transversalen Wimperreihen. Exopodit $40 \%$ kürzer als Endopodit, mit 2 terminalen und 3 Borsten am Außenrand; subterminal innen eine Wimpernreihe.

Beschreibung des $q_{1}$

Körperlänge 2,18 mm, 12 mal länger als die größte Breite.

Antenne I ohne Antennalorgan; Th. VIII ein kleines rundliches Läppchen mit winziger apikaler Spitze. Die übrigen Merkmale stimmen im Rahmen der Variationsbreite mit denen des $\hat{\sigma}$ überein.

\section{Variationen:}

1. Antenne I am 2. Glied mit 1 oder 2 ventro-medialen Borsten: am 3. Glied mit 2 oder 3 Innenrandborsten.

2. Exopodit des Th. VI $q$ dreigliedrig; Beborstung der Endo- und Exopoditen von Th. I-VII variabel.

3. Sympodit der Uropoden mit 11-12 Dornen insgesamt.

4. Furkà mit 6-7 Innenranddornen. 
Der neuen Gattung steht die Gattung Notobathynella Schminke, 1973 am nächsten. Afrobathynella gen. n. unterscheidet sich von ihr durch den Bau des Th. VIII $\hat{\delta}$ und des Antennalorgans sowie durch das Vorhandensein von Pleopodenresten. Ein ausführlicher Vergleich wird im Zusammenhang mit der Beschreibung weiterer Parabathynellidae aus Südafrika an anderer Stelle gegeben werden.

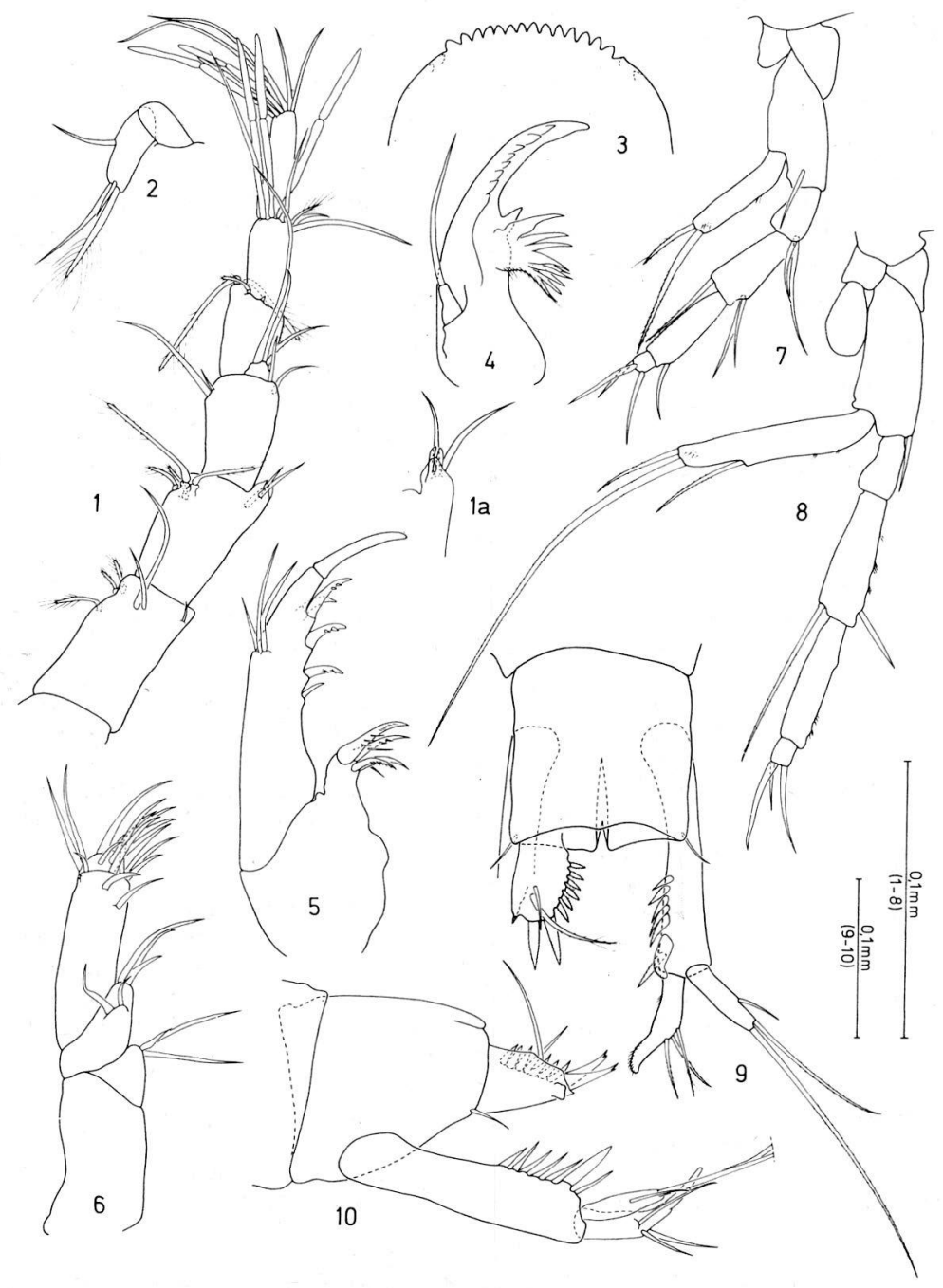

Tafel 4

Nunubathynella dimera gen. n., sp. n. $\hat{O}_{1}$ und $\hat{O}_{2}$

1. - Antenne I $\hat{\delta}_{1} ;$ la. - Antennalorgan $\hat{\delta}_{2}$ (von ventral); 2 . - Antenne II $\hat{\delta}_{1} ; 3$. - Labrum $\hat{\delta}_{1} ; 4$. Mandibel $\hat{\delta}_{1} ;$ 5. - Maxille I $\hat{\delta}_{1} ; 6$. - Maxille II $\hat{\delta}_{1} ; 7$. - Thorakopod I $\hat{\delta}_{1} ; 8$. - Thorakopod VII $\hat{\delta}_{1} ; 9$.Pleotelson $\hat{\delta}_{1}$ (dorsal); 10. - Pleotelson $\hat{\sigma}_{1}$ (lateral). 

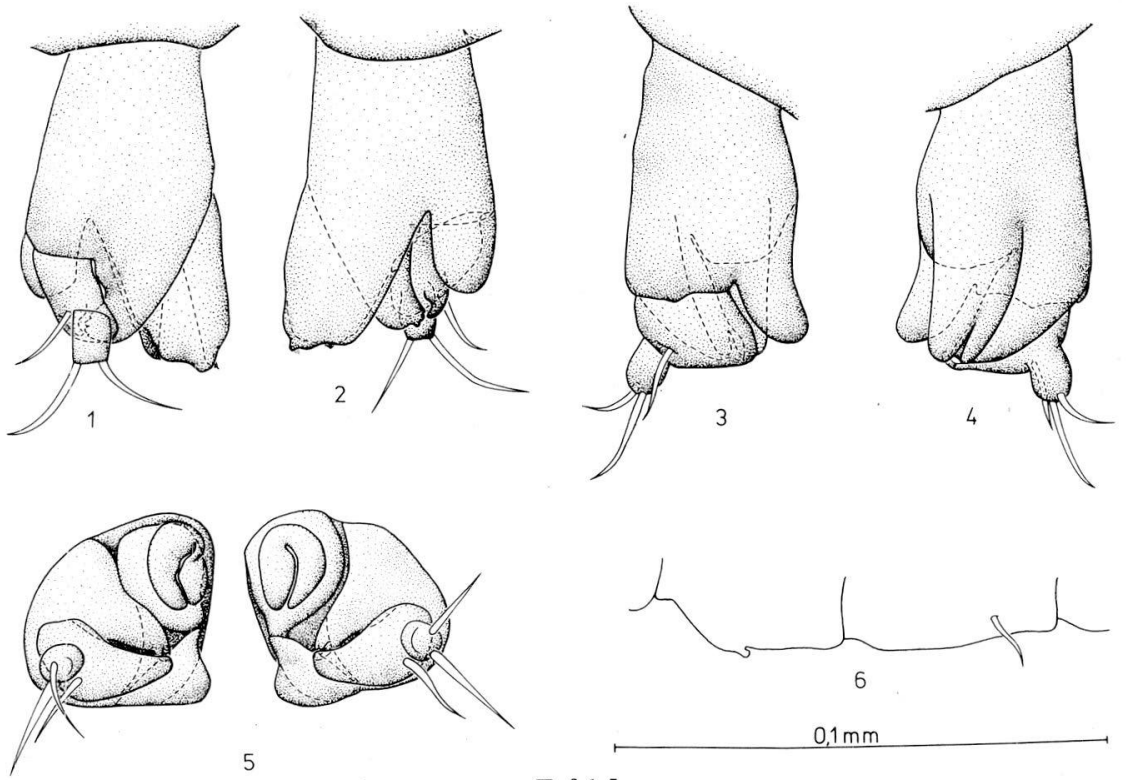

Tafel 5

Nunubathynella dimera gen. n., sp. n. $\hat{\delta}$, und $q$,

1. - Rechter Thorakopod VIII $\hat{\delta}_{1}$ (latero-extern); 2. - Derselbe (latero-intern); 3. - Derselbe (frontal von hinten); 4. - Derselbe (frontal von vorn); 5. - Beide Thorakopoden VIII $\delta$ । (apikal); 6. Thorakopod VIII und 1. Pleopod ${ }_{1}$.

Der neuen Gattung steht die Gattung Notobathynella Schminke, 1973 am nächsten. Afrobathynella gen. n. unterscheidet sich von ihr durch den Bau des Th. VIII und des Antennalorgans sowie clurch das Vorhandensein von Pleopodenresten. Ein ausführlicher Vergleich wird im Zusammenhang mit der Beschreibung weiterer Parabathynellidae aus Südafrika an anderer Stelle gegeben werden.

\section{Nunubathynella dimera gen. n.,sp. n.}

Derivatio nominis: nunu - in der Zulusprache: Tier, das versteckt lebt, so daß man es nicht sehen kann; unbekanntes Tier.

\section{Gattungsdiagnose:}

Th. VIII $\hat{\sigma}$ mit zweilappigem Penisanhang und dahinter einer caudalen Vorwölbung des Grundgliedes. Der Epipodit reicht mit seiner gezähnelten Spitze in den Raum zwischen diese beiden Anhänge und geht außen mit breiter Basis ohne Abgrenzung in das Grundglied über. Basipodit nach innen hin ebenfalls in eine Spitze auslaufend, die zum Epipoditen hin gebogen ist, neben dem Endopoditen mit einer Borste; Endopodit eingliedrig, mit 2 apikalen Borsten; kein Exopodit; Antenne II zweigliedrig, distales Glied mit 3 terminalen Borsten und 1 weiteren Borste auf halber Länge am Innenrand. Maxille I mit schlankem distalen Enditen, der in einer mächtigen Greifklaue endet, Innenrand zusätzlich mit 4 kleinen Klauen und 1 Borste. Maxille II viergliedrig, schlank. Exopoditen der Thorakopoden I-VII eingliedrig. 1. Pleopodenpaar in Form zweier Borsten vorhanden. Sympodit der Uropoden mit inhomonomem Dornenkamm, wobei der vorletzte Dorn kräftiger als die übrigen ist; Endopodit in Stilettform. Antenne I sechsgliedrig, mit komplexem Antennalorgan; Furka mit vielen Dornen.

Typusart: Nunubathynella dimera gen. n., sp. n.

Material: $\hat{\delta}_{1}$ (Holotyp, als Dauerpräparat auf 7 Objektträgern) sowie weitere $8 \hat{\delta} \hat{\delta}, 2 q q, 2$ juv.; Probe ZA-83L (vgl. Afrobathynella gen. n.); weitere Fundstellen: Probe ZA-64L vom 20.9. 
1973: 1 ऊ̂ं, 1 Q , 1 juv.; dieselbe Fundstelle wie ZA-83L; 1 Grabung im Mittelkies des Ufers von 85 $\mathrm{cm}$ Tiefe, $2,00 \mathrm{~m}$ vom Wasserlauf entfernt; Temperatur: $18^{\circ} \mathrm{C}$, Temperatur des oberirdischen Wassers: $18^{\circ} \mathrm{C}$; Begleitfauna: Nematoden, Oligochaeten, Acari, Collembolen, Insektenlarven, Cyclopiden, Harpacticiden, Ostracoden, Isopoden, Amphipoden.

Probe ZA-84L vom 8.8.1974: $q_{1}$ (als Dauerpräparat auf 6 Objektträgern) sowie weitere $5 q q$, $4 \hat{\delta} \delta$, 7 juv.; Baviaanskloof River an der Straße Patensie-Willowmore, $2 \mathrm{~km}$ von Studtis, Eastern Cape, South Africa; Fluß in mehrere Wasserarme aufgelöst, die sich, von Vegetation eingefaßt, über das ansonsten trockenliegende Flußbett schlängeln; 1 Grabung von $40 \mathrm{~cm}$ Tiefe im Mittelkies, 4,50 m neben einem der Wasserarme; Temperatur: $14,5^{\circ} \mathrm{C}$, Temperatur des oberirdischen Wassers: $15^{\circ} \mathrm{C}$; Begleitfauna: Nematoden, Cyclopiden, Harpacticien, Amphipoden, Bathybellidae, Leptobathynella spec.

Beschreibung des Typusexemplars: $\hat{\sigma} 1$

Mittelgroße Parabathynellidae von 1,56 mm Länge; 13 mal so lang wie die größte Breite; so hoch wie breit; Kopf $30 \%$ länger als breit.

Analoperculum medial leicht vorgebuchtet, aber nicht über den Rand des Pleotelsons hinausragend.

Furka fast doppelt so lang wie breit, mit 2 terminalen Dornen und 7 weiteren entlang des Innenrandes; von den 2 dorsolateralen Borsten ist die äußere 3 mal so lang wie die innere; Furkalorgan groß, lateral.

Antenne I sechsgliedrig; 30\% länger als der Kopf; Beborstung vgl. Tafel 4,1; 2. Glied mit komplexem Antennalorgan, das aus einem zweigipfligen Vorsprung besteht; auf jedem Gipfel mündet eine schnabelartige Öffnung; innen neben der Öffnung des dorsalen Gipfels befindet sich eine Borste.

Antenne II zweigliedrig; 85\% kürzer als Antenne I; weitere Einzelheiten vgl. Tafel 4,2.

Labrum mit 14 rundlichen Hauptzähnen.

Mandibel mit 8 Zähnen am distalen Teil der pars incisiva und einem Borstenlobus mit 8-9 Klauen; distale Klaue basal verdickt; die Borste des Palpus ist 3,5 mal länger als das Grundglied.

Maxille I vgl. Tafel 4,5.

Maxille II schlank, viergliedrig; mit spaltförmiger Öffnung am Außenrand des vorletzten an der Grenze zum distalen Glied; Borstenformel 3-5-8-7.

Thorakopoden von Th. I-V an Länge zunehmend, Th. VII etwa so lang wie Th. V, Th. VI kürzer; Th. II-VII mit je einem Epipoditen; Basis von Th. I-VII mit einer Innenrandborste; Exopoditen von Th. I-VII eingliedrig, der von Th. I mit 2 terminalen Borsten, die von Th. II-VII zusätzlich mit l ventralen Borste; Endopodit stets viergliedrig, Borstenformeln:

Th. I $2+0 / 2+1 / 1+0 / 3(1)$

Th. II-III $\quad 1+0 / 1+1 / 0+0 / 3(1)$

Th. IV-VII $\quad 0+0 / 1+1 / 0+0 / 3(1)$

Th. VIII mit gedrungenem Grundglied, das sich auf der Inneriseite nach vorn in einen zweilappigen Penisanhang verlängert und dahinter in einen rundlichen Anhang übergeht, der halb so lang wie der Penisanhang ist, und an den sich außen die Basis anschließt; außen geht das Grundglied ohne Abgrenzung in den Epipoditen über, der sich zuspitzt und nach innen in den Raum zwischen Penisanhang und caudalem Vorsprung des Grundgliedes reicht; die Spitze des Epipoditen ist mit Zähnchen besetzt. Basis nach innen ebenfalls spitz auslaufend und sich mit der Spitze zum Epipoditen hinwendend, so daß in Seitenansicht der Eindruck entsteht, als sei ein kleiner Exopodit vorhanden. Es persistiert aber nur der Endopodit, der 2 apikale Borsten trägt und neben dem an der Basis eine Borste inseriert. Beide Thorakopoden sind in der Mitte durch einen Zwischenraum getrennt.

1. Pleopodenpaar in Form zweier Borsten vorhanden.

Sympodit der Uropoden $6 \mathrm{mal}$ so lang wie breit, mit insgesamt 7 Dornen, von denen der vorletzte dicker und länger als die übrigen ist. Endopodit knapp halb so lang wie Sympodit, in Stilettform, innen mit 2 transversalen Wimpernreihen, außen neben dem Zahn mit 3 gleichlangen Borsten. Exopodit 30\% kürzer als Endopodit, mit 2 distalen Borsten und 1 weiteren Borste am Außenrand, innen subterminal eine kleine Wimpernreihe. 
Beschreibung des $q$

Körperlänge 1,40 mm; $14 \mathrm{mal} \mathrm{länger} \mathrm{als} \mathrm{die} \mathrm{größte} \mathrm{Breite;} \mathrm{Antenne} \mathrm{I} \mathrm{ohne} \mathrm{Antennalorgan;} \mathrm{Th.}$ VIII in Form eines kleinen rundlichen Läppchens vorhanden. Die übrigen Merkmale stimmen im Rahmen der Variationsbreite mit denen des $\hat{O}$ überein.

\section{Variationen:}

1. Antennalorgan auch noch mit einer 2. Borste unter der Öffnung des dorsalen Gipfels.

2. Labrum auch mit nur 12 Hauptzähnen.

3. Distaler Teil der pars incisiva der Mandibel mit 7-8 Zähnen, Borstenlobus mit 7-9 Klauen.

4. Sympodit der Uropoden mit 7-8 Dornen.

5. Analoperculum auch leicht über den Rand des Pleotelsons vorragend.

6. Furka mit 8-9 Dornen insgesamt.

Die neue Gattung steht Afrabathynella gen. n. nahe. Unterschiede vgl. p. 205.

Die Typen der beiden neuen Arten befinden sich vorläufig in der Sammlung des Verfassers.

Danksagungen: Diese Arbeit entstand mit dankenswerter Unterstützung durch die Deutsche Forschungsgemeinschaft. Herrn Prof. Dr. T. Erasmus danke ich für gastliche Aufnahme am Zoologischen Institut der Universität von Port Elisabeth, Südafrika, Herrn T. Hecht für seine Ratschläge und Hilfe auf den Exkursionsfahrten in die Umgebung von Port Elisabeth. Herrn Dr. W. Besch, Karlsruhe, gilt mein Dank für die Überlassung von Bathynellen-Material aus der Umgebung von Karlsruhe. Meinen Kollegen Herrn Dr. S. Lorenzen, Herrn Dr. P. Ohm und Herrn Dr. G. Weigmann danke ich für kritische Anmerkungen zum Manuskript dieser Arbeit, Frl. W. Röhe-Hansen, daß sie die Abbildungen druckfertig gemacht hat.

\section{ZUSAMMENFASSUNG}

Drei unterschiedliche Versuche der Revision dreier verschiedener Familien von Grundwasserkrebsen werden kritisch analysiert. Eingegangen wird auf die Bearbeitung der Copepodenfamilie Parastenocarididae durch Jakobi (1972), auf die Arbeiten von Serban und Serban et al. über die Bathynellidae (Bathynellacea, Syncarida) und auf die Revision der Parabathynellidae (Bathynellacea, Syncarida) durch Schminke (1973). Positive und negative Aspekte aller 3 Revisionsversuche werden diskutiert und Hinweise für die zukünftige Beschäftigung mit den jeweiligen Familien gegeben. Im Anhang werden 2 neue Gattungen der Familie Parabathynellidae, Afrobathynella gen. n. und Nunubathynella gen. n., aus dem Mesopsammal eines Flusses in der Nähe von Port Elisabeth (Südafrika) beschrieben.

\section{LITERATUR}

Delamare DeBOUTTEVILle, C. \& SERBAN, E. (1973) - A propos du genre Austrobathynella (Bathynellacea Malacostraca). - Livre du cinquantenaire de l'Institut de Spéologie "Emile Racovitza", Ed. Academiei R.S.R., Bucuresti 1973.

HENNIG, W. (1950) - Grundzüge einer Theorie der Phylogenetischen Systematik. Deutscher Zentralverlag, Berlin 1950.

ILLIES, J. (1970) - Die Gattung als ökologische Grundeinheit. — Faun. ökol. Mitt. 3: 369-372.

INGER, R. F. (1958) - Comments on the definition of genera. - Evolution 12: 370-384.

JAKOBI, H. (1954) - Biologie, Entwicklungsgeschichte und Systematik von Bathynella natans VEJD. - Zool. Jb. (Syst.) 83: 1-62.

(1972a) - Trends (Enp. P4 $O^{A}$ ) innerhalb der Parastenocarididen (Copepoda Harpacti-

coidea). - Crustaceana 22: 127-146. lien). - Stud. Neotrop. Faun. 7: 239-251.

MAYR, E. (1969) - Principles of Systematic Zoology. McGraw-Hill Book Company, New York 1969.

NOODT, W. (1965) - Natürliches System und Biogeographie der Syncarida (Crustacea, Malacostraca). - Gewäss. Abwäss. 37/38: 77-186. 
SCHLEE, D. (1971) - Die Rekonstruktion der Phylogenese mit Hennig's Prinzip. - Aufsätze Red. senckenberg. naturforsch. Ges. 20: 1-62.

SCHMINKE, H. K. (1972) - Hexabathynella halophila gen. n., sp. n. und die Frage nach der marinen Abkunft der Bathynellacea (Crustacea: Malacostraca). - Mar. Biol. 15: 282-287. (1973a) - Ökologische Aspekte der Gattungsgliederung der Familie Parabathynellidae (Bathynellacea, Malacostraca). - Z. zool. Syst. Evolutionsforsch. 11: 154-160.

(1973b) - Evolution, System und Verbreitungsgeschichte der Familie Parabathynellidae (Bathynellacea, Malacostraca). - Akad. Wiss. Lit. Mainz, Mikrofauna Meeresboden 24: 1-192.

SERBAN, E. (1972) - Bathynella (Podophallocarida, Bathynellacea). - Trav. Inst. Spéol. "Emile Racovitza" 11: 11-224.

(1973a) - Sur les problèmes de la taxonomie des Bathynellidae (Podophallocarida, Bathynellacea). - Livre du cinquantenaire de l'Institut de Spéologie "Emile Racovitza": 199-217, Ed. Academiei R.S.R., Bucuresti 1973.

(1973b) - Sur les Bathynellidae de l'Italie: 2. Sardobathynella cottarellii n. g. et n. sp. (Podophallocarida, Bathynellacea). - Fragm. Entomol. 9: 81-107.

, COINEAU, N. \& DELAMARE DEBOUTTEVILLE, C. (1972) - Recherches sur les Crustacés souterrains et mésopsammiques I. Les Bathynellacés (Malacostraca) des régions méridionales de l'Europe Occidentale. La sous-famille des Gallobathynellinae. Mém. Mus. Nat. Hist. Nat. Paris, N.S. (A), Zool., 75:7-107. 